\title{
Strategy to Accelerate or Augment the Antidepressant Response and for An Early Onset of SSRI Activity. Adjunctive Amisulpride to Fluvoxamine in Major Depressive Disorder
}

\author{
Hardoy Maria Carolina* and Carta Mauro Giovanni \\ Department of Public Health, Centre for Research and Clinical Practice in Mental Health, Iglesias, University of \\ Cagliari, Italy
}

\begin{abstract}
The topic of early response to antidepressant treatment has been extensively studied in major depressive disorder (MDD). We serendipitous observed an increase tolerability, a rapid response to therapy and an early onset of antidepressant fluvoxamine activity when associated with amisulpride in patients with major depressive disorder. The purpose of this study was to investigate our preliminary observations.
\end{abstract}

Keywords: Antidepressant, major depressive disorder, community survey, epidemiology, lifetime prevalence.

\section{INTRODUCTION}

One of the principal stakes of depression treatment are to accelerate and enhance the clinical effects of antidepressant drug [1].

The development of selective serotonin reuptake inhibitors (SSRIs) provided a major advancement in the treatment of depression. However, these drugs suffer from a variety of drawbacks, most notably a delay in the onset of efficacy [2].

The delay in the response of SSRIs has been investigated both in the field of clinical and preclinical research.

According to main observations $[3,4]$ the onset of action with SSRI agents depends on the activation of the 5HT-1A autoreceptors, which one tries to hinder by using drugs able to operate a selective blockade [5].

Although SSRIs block serotonin (5-hydroxytryptamine, 5-HT) reuptake rapidly, their therapeutic action is delayed. One hypothesis suggests that this delay in efficacy is due to a paradoxical decrease in serotonergic (5-HT) neuronal impulse flow and release, following activation of inhibitory presynaptic 5-HT1A autoreceptors, following acute administration of SSRIs. According to the hypothesis, efficacy is seen only when this impulse flow is restored following desensitization of 5-HT1A autoreceptors and coincident increases in postsynaptic 5-HT levels are achieved [2].

The onset of antidepressant action of SSRIs was attributed in part to this decrease in firing activity of serotonin neurons produced by the activation of raphe $5 \mathrm{HT} 1 \mathrm{~A}$ autoreceptors at the time of treatment initiation $[1,6,7]$.

The increase in synaptic 5-HT activates feedback mechanisms mediated by 5-HT1A (cell body) and 5-HT1B (terminal) autoreceptors, which, respectively, reduce the firing in 5-HT neurons and decrease the amount of 5-HT released per action potential resulting in attenuated 5-HT neurotransmis-

*Address correspondence to this author at the Viale Merello 22, Cagliari, Italy; Tel: +39 3338452928; E-mail: mgcarta @ tiscali.it sion. Long-term treatment desensitizes the inhibitory 5-HT1 autoreceptors, and 5-HT neurotransmission is enhanced. The time course of these events is similar to the delay of clinical action [8].

Clinical proof of this principal has been suggested in studies that found a significant augmenting effect when the beta-adrenergic/5-HT1A receptor antagonist was coadministered with SSRI treatment [2, 9].

Pindolol, an antagonist at somatodendritic pre-synaptic 5HT1A receptors has been investigated as a potential accelerator or potentialisator of antidepressant response [1].

The addition of pindolol, which blocks 5-HT1A receptors, to SSRI treatment decouples the feedback inhibition of 5-HT neuron firing and accelerates and enhances the antidepressant response $[6,7]$.

The neuronal circuitry of the 5-HT and norepinephrine (NE) systems and their connections to forebrain areas believed to be involved in depression has been dissected. The firing of 5-HT neurons in the raphe nuclei is driven, at least partly, by alpha1-adrenoceptor-mediated excitatory inputs from NE neurons. Inhibitory alpha2-adrenoceptors on the NE neuroterminals form part of a feedback control mechanism [10].

Results indicate that (-)pindolol potentiated the activation of postsynaptic 5-HT(1A) receptors resulting from 5-HT reuptake inhibition probably by blocking the somatodendritic 5-HT(1A) autoreceptor, but not its postsynaptic congener. These results support and extend previous findings providing a biological substratum for the efficacy of pindolol as an accelerating strategy in major depression [11].

In any case how such drugs as pindolol actually work is still controversial [12-14].

Gartside et al. have suggested that both (-)-tertatolol and (-)-penbutolol are superior to (+/-)pindolol in terms of enhancing the effect of an SSRI on extracellular 5-HT [9].

We serendipitous observed in patients with both dysthymia/major depression symptoms and, in open trial, 
with others presenting only major depressive disorder a decreased latency period in the action of SSRI when associated with amisulpride [15].

We therefore planned a large open trial with the concurrent administration of SSRI fluvoxamine, as a base antidepressant, and substituted benzamide amisulpride.

Although antidepressant medications are effective in about $50-70 \%$ of patients with major depressive disorder (MDD), they have a delayed onset of therapeutic effect. This latency on the onset of action is one of the current major limitations of these medications, in that it prolongs the impairments associated with depression, leaves patients vulnerable to an increased risk of suicide, increases the likelihood that a patient will prematurely discontinue therapy, and increases medical costs associated with severe depression [16].

SSRIs are increasingly used in the treatment of depression [17]. They have efficacy limitations, such as a 2-to 4week delay before the onset of symptom relief. They are well tolerated. The most frequently reported adverse event is nausea [18].

The use of SSRIs was suggested by the evaluation of large double-blind, randomized, controlled trials outlining fluvoxamine as effective as other SSRIs, but endowed with shorter therapeutic latency [19] and an earlier onset of its action when associated with pindolol rather than desipramine, thus proving ideal in the search of an optimized latency period.

The dopaminergic antidepressant action of substituted benzamides has been proposed, since the late 1970s, by several authors and extensively explored in preclinical experiments [20]. The class of substituted benzamides includes compounds able to modulate dopaminergic neurons selectively and specifically. The first synthetic substituted benzamide was sulpiride, which has been replaced in the clinic by the more modern amisulpride [21]. In Italy, the first marketing authorization obtained for the amisulpride, was with the sole indication of dysthymia and therefore a solid clinical experience exists in the use of substituted benzamides in mild forms of depression. Amisulpride is a substituted benzamide that, at low doses, selectively blocks D2 and D3 presynaptic dopamine receptors, enhancing dopaminergic transmission in frontal cortex and limbic areas. Many clinical studies versus placebo, tricyclic antidepressants and selective serotonin reuptake inhibitors showed amisulpride antidepressant effect, supporting its safety and rapid onset of action [22]. The proposed mechanism of action of substituted benzamides implies a selective modulation of the dopaminergic system in the mesocorticolimbic area, important for cognitive processing of internal and external cues, related to survival [20]. The compound is very selective for mesolimbic D2 and D3 receptors and, therefore, has a dual mechanism of action, which is associated with two different indications. At low doses $(50 \mathrm{mg})$, amisulpride preferentially blocks presynaptic autoreceptors, producing an increase in dopamine release, and therefore acting as a dopaminergic compound able to resolve the dopaminergic hypoactivity that characterizes depression. At higher doses (400-1.200 mg), the drug exerts its activity on postsynaptic $\mathrm{D} 3 / \mathrm{D} 2$ receptors located in the limbic region and prefrontal areas, producing selective dopaminergic inhibition, eliciting antipsychotic effects. Based on experimental data, amisulpride is a treatment of choice for dopaminergic transmission disorders, both in depression and in schizophrenia [21].

\section{MATERIALS AND METHODS}

A trial carried out on 20 women (mean age 51.3 years) with DSM-IV TR [23] diagnostic criteria for major depressive disorder and a Hamilton Depression Rating Scale (HDRS) [24-26] score higher than 20.

Exclusion factor was the age under 35 years.

Each patient was given fluvoxamine $(100 \mathrm{mg} /$ day $)$ and amisulpride $(50 \mathrm{mg} /$ day) throughout the 6 week trial.

Clinical symptoms were evaluated by using Hamilton Depression Rating Scale (HDRS) [24-26] at the end of week $1,2,3,6$.

\section{RESULTS}

HDRS score at baseline was $26.2 \pm 5.2$ and at the end of the trial the mean score was $9.6 \pm 3.8$. At the end of the week6 only one patient presented a HDRS score higher than 20 .

The ANOVA one way for repeated measures carried out on the basis of the HDRS score at baseline and at week 1,2, 3 and 6 stage expressed a statistically significant improvement of depressive symptoms ( $\mathrm{F}=4.5$; $\mathrm{DF} 19,80,4,76,99$; $\mathrm{P}<$ $0.00001)$. Table 1 shows the HDRS scores at the different evaluations of the trial.

The difference of the HDRS score reached the statistically significance between baseline and week 1 and then progressively increased.

Nineteen patients showed a clear improvement of depressive symptoms between the day 0 and 14. The HDRS item analysis demonstrated that the first therapeutic effect was the disappearance of the sleep depressive pattern. This effect was clear in 10 patients at the end of the first week.

None of the patients expressed significant side effects. Two patients complained excessive drowsiness.

\section{CONCLUSION}

Though methodologically limited (short sample and open trial) the study appears to suggest an increased SSRI tolerability (absence of nausea and vomiting) and a shorter latency period.

The first information is not surprising, considering the peculiar fluvoxamine and SSRI side effects related to gastric functions and nausea and the specific anti-nausea action of substituted benzamides.

On the other hand the earlier onset of SSRI activity defies easy interpretation. It is however important to remember that depressive symptomatology, at least in some cases, is mediated by final dopaminergic pathways, on which serotoninergic and adrenergic intermediate carriers may interfere [27].

The early onset of action on depressive sleep pointed out by this study seems to have a relationship (and to some extent seems to confirm) with a specific fluvoxamine effect on the sleep depressive pattern. This action appears to be a characteristic of this drug in comparison with other SSRIs [19]. 
Table 1. Hamilton Depression Rating Scale Scores at the Different Evaluations During the Trial

\begin{tabular}{|c|c|c|c|c|}
\hline Stage of Trial & HDRS Score & SD & $\begin{array}{c}\text { F ANOVA } \\
\text { Vs T0 (DF 1,38,39) }\end{array}$ & P \\
\hline \hline To & 26.2 & 2.9 & & 0.003 \\
\hline T1 & 21.9 & 5.4 & 9.38 & 0.002 \\
\hline T2 & 16.1 & 13.4 & 10.38 & $<0.0001$ \\
\hline T3 & 13.4 & 3.9 & 235.4 & $<0.0001$ \\
\hline
\end{tabular}

Adjunctive amisulpride to fluvoxamine indicate that they may be a potential strategy for an early onset of SSRI activity in the treatment of major depressive disorder. Further and more detailed reports are necessary to confirm these findings.

\section{AUTHORS' CONTRIBUTIONS}

$\mathrm{MCH}, \mathrm{MGC}$ conceived of the study, participated in its design and coordination, drafted the manuscript. MGC performed the statistical analysis. Both authors read and approved the final manuscript.

\section{REFERENCES}

[1] Brousse G, Schmitt A, Chereau I, Eschalier A, Dubray C, Llorca PM. Interest of the use of pindolol in the treatment of depression: review. Encephale 2003; 29(4 Pt 1): 338-50.

[2] Kinney GG, Taber MT, Gribkoff VK. The augmentation hypothesis for improvement of antidepressant therapy: is pindolol a suitable candidate for testing the ability of 5HT1A receptor antagonists to enhance SSRI efficacy and onset latency? Mol Neurobiol 2000; 21(3): 137-52.

[3] Blier P, Bergeron R, De Montigny C. Selective activation of postsynaptic 5HT-1A receptors induces rapid antidepressant response. Neuropsychopharmacology 1997; 16: 333-8.

[4] De Montigny C, Blier P. Augmentation strategies in treatment resistant depression: preclinical and clinical aspects. Eur Psychiatry 1998; 13(4): S60-6.

[5] Perez V, Gilaberte I, Faries D, Alvarez E, Artigas F. Randomized double blind placebo controlled trial of pindolol in combination with fluoxetine treatment. Lancet 1997; 349: 1594-7.

[6] Blier P. Neurobiological basis for the potentiation of antidepressant treatments by pindolol. Eur Neuropsychopharmacol 1998; 8(2): S96.

[7] Blier P, Bergeron R. The use of pindolol to potentiate antidepressant medication. J Clin Psychiatry 1998; 59 (Suppl 5): 16-23; discussion 24-5.

[8] Gardier AM, Malagi ŽI, Trillat AC, Jacquot C, Artigas F. Role of 5-HT1A autoreceptors in the mechanism of action of serotoninergic antidepressant drugs: recent findings from in vivo microdialysis studies. Fundam Clin Pharmacol 1996; 10(1): 16-27.

[9] Gartside SE, Clifford EM, Cowen PJ, Sharp T. Effects of (-)tertatolol, (-)-penbutolol and (+/-) pindolol in combination with paroxetine on presynaptic 5-HT function: an in vivo microdialysis and electrophysiological study. Br J Pharmacol 1999; 127(1): 14552.

[10] Blier P. Possible neurobiological mechanisms underlying faster onset of antidepressant action. J Clin Psychiatry 2001; 62 (Supp1 4): 7-11; discussion 37-40.
[11] BŽ•que JC, Blier P, de Montigny C, Debonnel G. Potentiation by (-)pindolol of the activation of postsynaptic 5-HT (1A) receptors induced by venlafaxine. Neuropsychopharmacology 2000; 23(3): 294-306.

[12] Artigas F. Pindolol augmentation: single or multiple targets in the brain. Eur Neuropsychopharmacol 1998; 8(2): S97.

[13] Zanardi R, Franchini L, Gasperini M, Lucca A, Smeraldi E, Perez J. Fluvoxamine and pindolol: a new and faster strategy in the treatment of delusional depression. Am Psychiatric Assoc Annu Meet Toronto 1998; 126.

[14] Cremers TI, Wiersma LJ, Bosker FJ, den Boer JA, Westerink BH, Wikstršm HV. Is the beneficial antidepressant effect of coadministration of pindolol really due to somatodendritic autoreceptor antagonism? Biol Psychiatry 2001; 50(1): 13-21.

[15] Carta MG, Hardoy MC, Murgia S, Hardoy MJ. Major depression: strategies for an early onset of SSRI activity: adjunctive amisulpride to fluvoxamine. Psychiatric Netw 1999; 2(1): 74-6.

[16] Gourion D. Antidepressants and their onset of action: a major clinical, methodological and pronostical issue. Encephale 2008; 34(1): 73-81.

[17] Deshauer D, Moher D, Fergusson D, Moher E, Sampson M, Grimshaw J. Selective serotonin reuptake inhibitors for unipolar depression: a systematic review of classic long-term randomized controlled trials. CMAJ 2008; 178(10): 1293-301.

[18] Katzman MA. Current considerations in the treatment of generalized anxiety disorder. CNS Drugs 2009; 23(2): 103-20.

[19] Van den Berg J. Fluvoxamine versus fluoxetine: a double blind randomized comparison in major depressive episode. Am Psychiatr Assoc Ann Meet Toronto 1998; 131.

[20] Pani L, Gessa GL: The substituted benzamides and their clinical potential on dysthymia and on the negative symptoms of schizophrenia. Mol Psychiatry 2002; 7: 247̃-53.

[21] Racagni G, Canonico PL, Ravizza L, Pani L, Amore M. Consensus on the use of substituted benzamides in psychiatric patients. Neuropsychobiology 2004; 50: 134-43.

[22] Torta R, Berra C, Binaschi L, Borio R. Amisulpride in the shortterm treatment of depressive and physical symptoms in cancer patients during chemotherapies. Support Care Cancer 2007; 15(5): $539-46$.

[23] American Psychiatric Association. Diagnostic and Statistical Manual of Mental Disorders. $4^{\text {th }}$ ed. Text Revision (DSM IV TR), USA 2000.

[24] Hamilton M. A rating scale for depression. J Neurol Neurosurg Psychiatry 1960; 23: 56-62.

[25] Hamilton M. Development of a rating scale for primary depressive illness. Br J Soc Clin Psychol 1967; 6(4): 278-96.

[26] Williams JB. A structured interview guide for the Hamilton depression rating scale. Arch Gen Psychiatry 1988; 45(8): 742-47.

[27] Serra G, Gessa GL. Role of mesolimbic dopamine system in the mechanism of action of antidepressant. Proc XXII CINP Copenhagen Pharmacol Toxicol 1992; 71: 72-85.

(C) Carolina and Giovanni; Licensee Bentham Open.

This is an open access article licensed under the terms of the Creative Commons Attribution Non-Commercial License (http: //creativecommons.org/licenses/by-nc/3.0/) which permits unrestricted, non-commercial use, distribution and reproduction in any medium, provided the work is properly cited. 